Internat. J. Math. \& Math. Sci.

Vol. 22, No. 2 (1999) 349-365

S 0161-1712<99>22349-6

(c) Electronic Publishing House

\title{
MATHEMATICAL APPROACH AND NUMERICAL ANALYSIS TO THE FUNDAMENTAL SOLUTIONS METHOD OF DIRICHLET PROBLEM
}

\author{
TETSUO INOUE
}

(Received 31 May 1996 and in revised form 7 July 1997)

\begin{abstract}
Potentially theoretical schemes in the fundamental solutions method will be proposed for Dirichlet problems of unbounded and bounded Jordan domains. The asymptotic theorem on extremal weighted polynomials will play fundamental roles to introduce a new scheme and to determine the distribution of charge points. Typical examples of the method will show that the numerical results of higher accuracy than those of the the conventional one can be obtained.
\end{abstract}

Keywords and phrases. Extremal weighted polynomial, weak convergence, normalized counting measure on zeros, fundamental solutions method, Dirichlet problems, charge points.

1991 Mathematics Subject Classification. 30E10, 41A10, 65E05.

1. Introduction. The fundamental solutions method (or charge simulation method) has been applied to the problem in electrical engineering, numerical conformal mappings [2, 9] and Dirichlet problems [12, 13, 14, 20, 21, 22, 23, 24, 26]. The principle of the method is the approximation of the solution by a linear combination of logarithmic potentials. Though the method requires only solving a system of simultaneous linear equations, it is possible to get a rather precise solution for boundary problems with respect to domains bounded by smooth curves.

In this paper, we study the fundamental solutions method for Dirichlet problems of unbounded and bounded Jordan domains.

The method established in this paper has the following characteristic: the new scheme and the distribution of charge points are theoretically proposed using the asymptotic theorems $[4,5,7,6,8,10,11,9,16,17,19,18]$ on extremal weighted polynomials and their zeros.

Applying the method to typical examples, we will show that numerical results of higher accuracy than those of conventional ones can be obtained.

The theoretical consideration for the fundamental solutions method of unbounded Jordan domains will perhaps first appear in this paper.

2. Definition. In this section, we describe the notions of weighted polynomials (shortly $w$-polynomials) and weighted capacity ( $w$-capacity) introduced by the author [5] and Mhaskar-Saff [17, 19], respectively. The definitions of normalized counting measures and the weak convergence are also shown. 
Let $D$ denote an unbounded domain whose boundary is a Jordan curve $\gamma$. Without loss of generality, we assume that $D$ contains $\infty$ and 0 in its interior and exterior, respectively.

Let $w=w(z)$ be an arbitrary, continuous, positive function defined on $\gamma$. For each integer $n \geq 1$, we let $P_{n, w}$ denote the class of all polynomials of the form

$$
p_{n, w}(z)=\prod_{i=1}^{n}\left[\left(z-z_{n, i}\right) w(z) w\left(z_{n, i}\right)\right],
$$

which we call $w$-polynomials of degree $n$.

Let $M(\gamma)$ denote the class of all positive unit Borel measures whose support is $\gamma$. We define the $w$-energy of $\sigma \in M(\gamma)$ and the $w$-capacity by

$$
I_{w}(\sigma)=\iint \log [|z-t| w(z) w(t)] d \sigma(z) d \sigma(t)
$$

and

$$
\operatorname{cap}(w, \gamma)=\exp \left(V_{w}\right)
$$

respectively, where

$$
V_{w}=V(w, \gamma)=\sup _{\sigma \in M(\gamma)} I_{w}(\sigma) .
$$

We note that the notions of $w$-energy and $w$-capacity were introduced in $[17,19]$.

Throughout the remainder of this paper, we assume that every $p_{n, w}(z)$ has all the zeros on $\gamma$. Though it seems that the assumption is rather strong, it is the key point to establish the new fundamental solutions method in this paper. It depends on uniformly distributed points (which are defined in Section 3) being on the boundary $\gamma$.

Since we have defined the $w$-polynomials by (2.1), it seems that $w(z)$ must be defined in the whole complex plane. However, we will soon understand why the assumption is sufficient in this paper.

Moreover, the form (2.1) instead of

$$
q_{n, w}(z)=\prod_{i=1}^{n}\left[\left(z-z_{n, i}\right) w\left(z_{n, i}\right)\right],
$$

(which would require $w(z)$ to be defined only on $\gamma$ ) has the advantage that the inequality

$$
\limsup _{n \rightarrow \infty}\left\|p_{n, w}(z)\right\|_{\gamma}^{1 / n} \leq \operatorname{cap}(w, \gamma)
$$

may hold, where $\left\|p_{n, w}(z)\right\|_{\gamma}$ is the sup norm on $\gamma$.

Let $\mu_{w} \in M(\gamma)$ be an extremal measure such that

$$
I_{w}\left(\mu_{w}\right)=V_{w} .
$$

The existence and the uniqueness of $\mu_{w}$ were shown in [19, Thm. 3.1(b)]. We assume that $S_{w}=\gamma$, where $S_{w}$ is support of $\mu_{w}$.

Lastly, we show the notions of the normalized counting measure on the zeros and 
the weak convergence as follows: for $w$-polynomials $p_{n, w}(z)$ of degree $n$, the discrete unit measure defined on compact sets in the complex plane $C$ with mass $1 / n$ at each zero of $p_{n, w}(z)$ will be denoted by $\mu_{n, w}=\mu\left(p_{n, w}\right)$. It will be called the normalized counting measure on the zeros of $p_{n, w}(z)$. If $p_{n, w}(z)$ has multiple zeros, the obvious modification will be considered.

The weak convergence of $v_{n}$ to $v$ as $n \rightarrow \infty$ will be defined by

$$
\lim _{n \rightarrow \infty} \int f d v_{n}=\int f d v
$$

for every continuous function in the complex plane $C$ with compact support.

3. Fundamental lemma. In approximation theory, the asymptotic behavior of extremal polynomials has been studied by a lot of authors $[4,5,7,6,8,10,11,9$, $16,17,19,18]$. In this section, we present the Fundamental Lemma on extremal $w$ polynomials that plays an important role establishing the new fundamental solutions method.

FUNDAMENTAL LEMMA. The necessary and sufficient condition that

$$
\lim _{n \rightarrow \infty}\left|\prod_{i=1}^{n}\left(z-z_{n, i}\right) w\left(z_{n, i}\right)\right|^{1 / n}=\exp \left\{\int \log [|z-t| w(t)] d \mu_{w}(t)\right\}
$$

holds uniformly on every compact subset of $D$ is

(A) $\mu_{n, w}$ converges weakly to $\mu_{w}$ as $n \rightarrow \infty$, where $\mu_{n, w}=\mu\left(p_{n, w}\right)$ is the normalized counting measure of $p_{n, w}=\prod_{i=1}^{n}\left[\left(z-z_{n, i}\right) w\left(z_{n, i}\right)\right]$.

Furthermore, if condition (A) is satisfied, the equality

$$
\frac{\exp \left\{\int \log [|z-t| w(t)] d \mu_{w}(t)\right\}}{\operatorname{cap}(w, \gamma)}=\frac{1}{w(z)}
$$

holds q.e. on $\gamma$ (we say that a property holds q.e. on $\gamma$ if the subset $\gamma^{\prime}$ of $\gamma$ where it does not hold has capacity zero).

Here, we will sketch the proof of Fundamental Lemma. With the above notation we state a well-known theorem on the zeros distribution of extremal $w$-polynomials which has been established in [17, p. 88-89].

LEMMA 3.1. Let

$$
p_{n, w}(z)=\prod_{i=1}^{n}\left[\left(z-z_{n, i}\right) w(z) w\left(z_{n, i}\right)\right]
$$

be $w$-polynomials of degree $n$ and let the equality

$$
\lim _{n \rightarrow \infty}\left|p_{n, w}(z)\right|^{1 / n}=\exp \left\{g_{w}(z)\right\}
$$

holds uniformly on every compact subset of $D$, where

$$
g_{w}(z)=\int \log [|z-t| w(z) w(t)] d \mu_{w}(t) .
$$

Then, $\mu_{n, w}$ converges weakly to $\mu_{w}$ as $n \rightarrow \infty$. 
In fact, Lemma 3.1 has been proved for the case when $\gamma$ lies on the real axis and $w$-polynomials

$$
s_{n, w}(z)=\prod_{i=1}^{n}\left[\left(z-z_{n, i}\right) w(z)\right]
$$

in [17]. Since the proof for the case of Lemma 3.1 is similar, we will omit it. Since the equalities (3.1) and (3.4) are equivalent, it has been shown that (A) is the necessary condition that (3.1) holds uniformly on every compact subset of $D$.

The following theorem has recently been established by the author. It is easily shown using [19, Cor. 4.2] and so is omitted.

LEMMA 3.2. Let $\mu_{n, w}$ converge weakly to $\mu_{w}$ as $n \rightarrow \infty$ and let $\gamma$ be of positive capacity at each point (i.e., $\operatorname{cap}(\{\zeta \in \gamma ;|z-\zeta|<\delta\})>0$ for every $z \in \gamma$ and every $\delta>0$ ). Then, we have

$$
\limsup _{n \rightarrow \infty}\left\|p_{n, w}(z)\right\|_{\gamma}^{1 / n} \leq \operatorname{cap}(w, \gamma)
$$

Since $\gamma$ is assumed to be a Jordan curve, it is of positive capacity at each point. Considering Lemma 3.2, we have the inequality (3.7) when the condition (A) is satisfied. Here, we note that

$$
\limsup _{n \rightarrow \infty}\left\|p_{n, w}(z)\right\|_{\gamma}^{1 / n} \leq \operatorname{cap}(w, \gamma)
$$

implies

$$
\lim _{n \rightarrow \infty}\left\|p_{n, w}(z)\right\|_{\gamma}^{1 / n}=\operatorname{cap}(w, \gamma) .
$$

This follows from the result [19, Thm. 2.1(e)] and the definition of $\operatorname{cap}(w, \gamma)$. More precisely, Theorem 2.1(e) says that the inequality

$$
\int \log |z-t| d \mu_{w}(t)+Q_{w}(z) \geq V_{w}-\int Q_{w}(t) d \mu_{w}(t)
$$

holds for all $z \in \gamma$, where $Q_{w}(z)=\log w(z)$.

Therefore, from the definition of $\operatorname{cap}(w, \gamma)$ and (3.10), we have

$$
\int \log [|z-t| w(z) w(t)] d \mu_{w}(t) \geq V_{w}
$$

for all $z \in \gamma$, which implies

$$
\left\|p_{n, w}(z)\right\|_{\gamma}^{n} \geq \operatorname{cap}(w, \gamma)
$$

Combining (3.8) and (3.12), we have the equality (3.9).

Under the assumption mentioned above, we state a lemma which was verified in [5].

LEMMA 3.3. Let

$$
p_{n, w}(z)=\prod_{i=1}^{n}\left[\left(z-z_{n, i}\right) w(z) w\left(z_{n, i}\right)\right]
$$


be w-polynomials of degree $n$ satisfying the conditions (3.14) and (3.15) below

$$
\begin{gathered}
\limsup _{n \rightarrow \infty}\left\|p_{n, w}(z)\right\|_{\gamma}^{1 / n} \leq \operatorname{cap}(w, \gamma) . \\
\liminf _{n \rightarrow \infty} \frac{1}{n} \sum_{i=1}^{n} \log w\left(z_{n, i}\right) \geq \int \log w(t) d \mu_{w}(t) .
\end{gathered}
$$

Then, there holds the equality

$$
\lim _{n \rightarrow \infty}\left|p_{n, w}(z)\right|^{1 / n}=\exp \left\{g_{w}(z)\right\}
$$

uniformly on every compact subset of $D$.

We note that if (3.16) holds uniformly on every compact subset of $D$, the property (3.15) follows without inf and with equality. It is easily shown by letting $z$ tend to infinity in (3.16).

If $\mu_{n, w}$ converges weakly to $\mu_{w}$ as $n \rightarrow \infty$, from the definition of the weak convergence we have the equalities

$$
\lim _{n \rightarrow \infty} \frac{1}{n} \sum_{i=1}^{n} \log w\left(z_{n, i}\right)=\lim _{n \rightarrow \infty} \int \log w(t) d \mu_{w, n}(t)=\int \log w(t) d \mu_{w}(t) .
$$

Therefore, Lemma 3.2 and Lemma 3.3 imply that (A) is the sufficient condition that (3.1) holds uniformly on every compact subset of $D$. Thus, the first part of Fundamental Lemma has been proved.

Next, we sketch the proof of (3.2). [19, Thm. 2.1(d)] says that

$$
\int \log |z-t| d \mu_{w}(t)+Q_{w}(z) \leq V_{w}-\int Q_{w}(t) d \mu_{w}(t)
$$

holds for q.e. $z \in \gamma$.

Combining (2.3), (3.10), and (3.18), it is easily shown that (3.2) holds for q.e. $z \in \gamma$. We will show here two extremal $w$-polynomials which satisfy condition (A), which means that the equality

$$
\lim _{n \rightarrow \infty}\left|\prod_{i=1}^{n}\left[\left(z-z_{n, i}\right) w\left(z_{n, i}\right)\right]\right|^{1 / n}=\exp \left\{\int \log [|z-t| w(t)] d \mu_{w}(t)\right\}
$$

holds uniformly on every compact subset of $D$.

For each integer $n \geq 1$, let $Q_{n, w}$ be a set of $w$-polynomials

$$
q_{n, w}(z)=\prod_{i=1}^{n}\left[\left(z-z_{n, i}\right) w(z) w\left(z_{n, i}\right)\right],
$$

where the zeros $\left\{z_{n, i}\right\}_{i=1}^{n}$ lie on the boundary $\gamma$ of $D$.

Let $q_{n, w}^{*}(z)$ be a polynomial such that

$$
\left\|q_{n, w}^{*}(z)\right\|_{\gamma}=\inf _{q_{n, w} \in Q_{n, w}}\left\|q_{n, w}(z)\right\|_{\gamma} .
$$


The existence of $q_{n, w}^{*}(z)$ is easily proved by the usual method. Then, $q_{n, w}^{*}(z)$ is called a $w$-Chebyshev polynomial with zeros on $\gamma$. It is known that it satisfies the condition (A) [7].

To show another polynomial satisfying condition (A), we introduce the definition of $w$-transfinite diameter. For each integer $n \geq 2$, let

$$
\begin{aligned}
\delta_{n}(w, \gamma) & =\sup _{z_{n, 1}, \ldots, z_{n, n} \in \gamma}\left\{\prod_{1 \leq i<j \leq n}\left[\left|z_{n, i}-z_{n, j}\right| w\left(z_{n, i}\right) w\left(z_{n, j}\right)\right]\right\}^{2 /(n(n-1))} \\
& =\left\{\prod_{1 \leq i<j \leq n}\left[\left|z_{n, i}^{*}-z_{n, j}^{*}\right| w\left(z_{n, i}^{*}\right) w\left(z_{n, j}^{*}\right)\right]\right\}^{2 /(n(n-1))} .
\end{aligned}
$$

The sequence $\left\{\delta_{n}(w, \gamma)\right\}_{n=1}^{\infty}$ converges and

$$
\tau(w, \gamma)=\lim _{n \rightarrow \infty} \delta_{n}(w, \gamma)
$$

is called $w$-transfinite diameter of $\gamma$. It is said that $\left\{z_{n, i}^{*}\right\}_{i=1}^{n}$ are $w$-Fekete points. It is then known that $w$-polynomials

$$
k_{n, w}(z)=\prod_{j=1}^{n}\left[\left(z-z_{n, j}^{*}\right) w(z) w\left(z_{n, j}^{*}\right)\right]
$$

satisfy condition (A) [16].

We will present a polynomial with the extremal points $\left\{z_{n, i}\right\}_{i=1}^{n}$ that satisfies (3.9) with $w(z)=1$. Here, we review the definition of Fejér points.

For a compact set $K$, let the conformal mapping

$$
\varphi(w)=d w+d_{0}+\frac{d_{1}}{w}+\cdots, \quad d>0
$$

from $\{w ;|w|>1\}$ onto $K^{c}$ have a continuous extension to $\{w ;|w| \geq 1\}$. Then, the points

$$
z_{n, k}=\varphi\left(e^{2 \pi i(k-1) / n}\right), \quad(k=1,2, \ldots, n)
$$

are called Fejér points of order $n$ on $K$.

Let $p_{n}(z)$ be a polynomial whose zeros are Fejér points. It is known [3, 25] that $p_{n}(z)$ satisfies

$$
\lim _{n \rightarrow \infty}\left\|p_{n}(z)\right\|_{K}^{1 / n}=\operatorname{cap}(K) .
$$

Therefore, from Lemma 3.3 the equality

$$
\lim _{n \rightarrow \infty}\left|\prod_{i=1}^{n} p_{n}(z)\right|^{1 / n}=\exp \left\{\int \log [|z-t|] d \mu_{1}(t)\right\}
$$

holds uniformly on every compact subset of $D$.

If condition (3.9) is satisfied for $w(z)=1$, the zeros $\left\{z_{n, i}\right\}_{i=1}^{n}$ of $p_{n}(z)$ are called to be uniformly distributed on $\gamma$ [3]. 
4. New scheme. Let $D$ and $D_{1}\left(D \subset D_{1}\right)$ be unbounded Jordan domains with $\partial D=\gamma$ and $\partial D_{1}=\gamma_{1}$. Also in this section, we assume that both of $D$ and $D_{1}$ contain $\infty$ and 0 in their interiors and exteriors, respectively.

Let the function $H(z)$ be harmonic in $D_{1}$, where $H(z)=h(z)$ and $h_{1}(z)$ on $\gamma$ and $\gamma_{1}$, respectively. Then, we apply Fundamental Lemma to the domain $D_{1}$ and let

$$
w_{1}(z)=\exp \left\{-\left(h_{1}(z)+\log |z|\right)\right\}=\frac{\exp \left\{-h_{1}(z)\right\}}{|z|} .
$$

When the points $\left\{z_{n, i}\right\}_{i=1}^{n}$ on $\gamma_{1}$ satisfying condition (A) are determined, the equalities

$$
H^{*}(z)=\log \lim _{n \rightarrow \infty} \frac{\prod_{i=1}^{n}\left|\left(z-z_{n, i}\right) w_{1}\left(z_{n, 1}\right)\right|^{1 / n}}{\operatorname{cap}\left(w_{1}, \gamma_{1}\right)}=\int \log \frac{|z-t| w_{1}(t)}{\operatorname{cap}\left(w_{1}, \gamma_{1}\right)} d \mu_{w_{1}}(t)
$$

hold uniformly on every compact subset of $D_{1}$, which follows from Fundamental Lemma.

Since $\gamma$ is a compact set in $D_{1}$, the convergence is uniform on $\gamma$. Furthermore, from (3.2)

$$
\frac{\exp \left\{\int \log \left[|z-t| w_{1}(t)\right] d \mu_{w_{1}}(t)\right\}}{\operatorname{cap}\left(w_{1}, \gamma_{1}\right)}=\frac{1}{w_{1}(z)}
$$

holds q.e. on $\gamma_{1}$. Combining (4.1), (4.2), and (4.3), the function

$$
H_{1}(z)=H^{*}(z)-\log |z|
$$

satisfies $H_{1}(z)=h_{1}(z)$ q.e. on $\gamma_{1}$. Since

$$
\lim _{z \rightarrow \infty} H_{1}(z)=\int \log \frac{w_{1}(t)}{\operatorname{cap}\left(w_{1}, \gamma_{1}\right)} d \mu_{w_{1}}(t)
$$

is finite, $H_{1}(z)$ is harmonic in $D_{1} \cup \infty[15,25]$.

Applying generalized Maximun Principle (two harmonic functions with q.e. same boundary values are equal to each other in the domain $[15,25])$ for the function $H(z)-$ $H_{1}(z)$, we obtain the equality $H(z)=H_{1}(z)$ in $D_{1} \cup \infty$.

Let $h(z)$ be a given function which is continuous on $\gamma$. The above argument suggests the following algorithm for the fundamental solutions method of Dirichlet problem (that is, to find the function $H(z)$ harmonic in $D \cup\{\infty\}$ such that $H(z)=h(z)$ on $\gamma$ ).

Algorithm 4.1. The approximation $H_{n}(z)$ of $H(z)$ is obtained as follows

(4a) The charge points are chosen on $\gamma_{1}$ such that condition (A) is satisfied.

(4b) The charge at every charge point is assumed to be $1 / n$.

(4c) The approximation $H_{n}(z)$ is represented as

$$
H_{n}(z)=H(\infty)+\frac{1}{n} \sum_{i=1}^{n} \log \left|1-\frac{z_{n, i}}{z}\right|
$$

for a sufficient large $n$. 
By Fundamental Lemma, the approximation $H_{n}(z)$ converges uniformly to $H(z)$ on $\bar{D}=D \cup \gamma($ not on compact sets of $D)$.

Though the numerical solution of Dirichlet problem is theoretically obtained by the above fundamental solutions method using (4a), (4b), and (4c), it is generally difficult to find the charge points satisfying condition (A) and $H(\infty)$ is not known.

Therefore, we propose the following Algorithm 4.2. The charge points are chosen by the same method as the conventional one. For example, the charge points for the domain $D=\{z ;|z|>1\}$ are uniformly distributed on a circle $\gamma_{1}=\{z ;|z|=r, r<1\}$. The collocation points are analogously chosen on $\gamma$.

Algorithm 4.2. The approximation $H_{n}^{*}(z)$ of $H(z)$ is obtained as follows

(4e) Let the charge points $\left\{z_{n, i}\right\}_{i=1}^{n}$ and the collocation points $\left\{\zeta_{n, i}\right\}_{i=1}^{n}$ be appropriately chosen on $\gamma_{1}$ and $\gamma$, respectively.

(4f) When $\alpha_{i}(i=0,1,2, \ldots, n)$ are the solution of a system of simultaneous linear equations

$$
\begin{gathered}
\alpha_{0}+\sum_{i=1}^{n} \alpha_{i} \log \left|1-\frac{z_{n, i}}{\zeta_{n, k}}\right|=h\left(\zeta_{n, k}\right), \quad(k=1,2, \ldots, n), \\
\alpha_{1}+\alpha_{2}+\cdots+\alpha_{n}=1,
\end{gathered}
$$

the charges at $\left\{z_{n, i}\right\}_{i=1}^{n}$ are given by $\left\{\alpha_{i}\right\}_{i=0}^{n}$.

(4g) The approximation $H_{n}^{*}(z)$ is represented by

$$
H_{n}^{*}(z)=\alpha_{0}+\sum_{i=1}^{n} \alpha_{i} \log \left|1-\frac{z_{n, i}}{z}\right|
$$

for a sufficient large $n$.

If the charge points and the collocation points are "theoretically" chosen, we suppose that the approximations

$$
\alpha_{0} \simeq H(\infty), \quad \alpha_{i} \simeq \frac{1}{n}, \quad(i=1,2, \ldots, n)
$$

would hold.

The numerical experiments in Section 5 for some examples show that the approximations hold with high accuracy.

Now, we consider the case when $D$ is a "bounded" Jordan domain containing 0 and $\infty$ in its interior and exterior, respectively. Using the transformation $\zeta=1 / z$, we propose the new scheme (to be called Inoue-Scheme) corresponding to (4.9).

Using the scheme, Algorithm 4.2 is translated to the following one for a bounded Jordan domain

Algorithm 4.3. The approximation $H_{n}(z)$ of $H(z)$ is obtained as follows

$\left(4 \mathrm{e}^{\prime}\right)$ Let the charge points $\left\{z_{n, i}\right\}_{i=1}^{n}$ and the collocation points $\left\{\zeta_{n, i}\right\}_{i=1}^{n}$ be appropriately chosen on $\gamma_{1}$ and $\gamma$, respectively. 
$\left(4 \mathrm{f}^{\prime}\right)$ When $\alpha_{i}(i=0,1,2, \ldots, n)$ are the solution of a system of simultaneous linear equations

$$
\begin{gathered}
\alpha_{0}+\sum_{i=1}^{n} \alpha_{i} \log \left|1-\frac{\zeta_{n, k}}{z_{n, i}}\right|=h\left(\zeta_{n, k}\right), \quad(k=1,2, \ldots, n), \\
\alpha_{1}+\alpha_{2}+\cdots+\alpha_{n}=1,
\end{gathered}
$$

the charges at $\left\{z_{n, i}\right\}_{i=1}^{n}$ are given by $\left\{\alpha_{i}\right\}_{i=0}^{n}$.

$\left(4 \mathrm{~g}^{\prime}\right)$ The approximation $H_{n}(z)$ is represented by

$$
H_{n}(z)=\alpha_{0}+\sum_{i=1}^{n} \alpha_{i} \log \left|1-\frac{z}{z_{n, i}}\right|
$$

for a sufficient large $n$.

Note the difference of the schemes of approximations between (4.9) and (4.13). If the charge points and the collocation points are "theoretically" chosen, we suppose that the approximations

$$
\alpha_{0} \simeq H(0), \quad \alpha_{i} \simeq \frac{1}{n}, \quad(i=1,2, \ldots, n)
$$

would hold also for bounded Jordan domains.

The numerical experiments for some examples in Section 5 show that the approximations hold with high accuracy.

Compare Inoue-Scheme to the conventional scheme (Conv-Scheme) $[12,13,14,24$, 26] and Murota-Scheme [21, 22]

$$
H_{n}(z)=\sum_{i=1}^{n} \alpha_{i} \log \left|z-z_{n, i}\right|
$$

and

$$
H_{n}(z)=\alpha_{0}+\sum_{i=1}^{n} \alpha_{i} \log \left|z-z_{n, i}\right|
$$

with

$$
\alpha_{1}+\alpha_{2}+\cdots+\alpha_{n}=0
$$

respectively.

Inoue-Scheme and Murota-Scheme have the advantage satisfying the following (called "an invariant property")

$$
z \rightarrow a z, \quad z_{n, i} \longrightarrow a z_{n, i}, \quad H(z) \longrightarrow H(z)+b
$$

implies

$$
H_{n}(z) \longrightarrow H_{n}(a z), \quad H_{n}(z) \longrightarrow H_{n}(z)+b,
$$

where $a(\neq 0), b$ are constant.

The property for the scheme is mathematically and physically natural in the sense that the approximations remain invariant with respect to trivial affine transformations. 
Though Murota-Scheme has the property under the condition (4.17), Inoue-Scheme (4.9) and (4.13) have it without any condition.

Applying Inoue-Scheme to typical examples, we will show that the numerical results of higher accuracy than those of conventional ones can be obtained.

We remark that Murota-Scheme is a special case of Inoue-Scheme, when $D$ is a disk with the center at the origin and the charge points are distributed on a circle with center at the origin. It depends on the equalities

$$
\begin{aligned}
\alpha_{0}+\sum_{i=1}^{n} \alpha_{i} \log \left|z-z_{n, i}\right| & =\alpha_{0}+\sum_{i=1}^{n} \alpha_{i} \log \left|z_{n, i}\right|+\sum_{i=1}^{n} \alpha_{i} \log \left|1-\frac{z}{z_{n, i}}\right| \\
& =\alpha_{0}+\sum_{i=1}^{n} \alpha_{i} \log \left|1-\frac{z}{z_{n, i}}\right|
\end{aligned}
$$

because $\log \left|z_{n, i}\right|=$ constant and (4.17) is satisfied.

5. Numerical example. This section deals with some numerical experiments for a disk $D=\{z:|z|<1\}$ with the boundary $\gamma=\{z ;|z|=1\}$ in order to estimate the accuracy of errors of the approximations by Conv-Scheme, Murota-Scheme and InoueScheme. More precisely, we consider a harmonic function

$$
H(z)=\log \left|\frac{z-3}{2}\right|
$$

in $D=\{z:|z|<1\}$.

Let

$$
\gamma_{l}=\left\{z ;\left|\frac{z-3}{2 z}\right|=l\right\}
$$

and

$$
\Gamma_{l}=\{z ;|z|=l\} .
$$

The charge points and the collocation points are chosen there. Then, the approximations $H_{n}(z)$ of $H(z)$ are obtained by the fundamental solutions method with Algorithm $4.1,4.2$, and 4.3 and the errors $H_{n}(z)-H(z)$ are estimated.

(5a) Using Inoue-Scheme (4.11) and (4.12), the charges will first be obtained when the charge points satisfy condition (A).

The charge points $\left\{z_{n, i}\right\}_{i=1}^{n}$ on $\gamma_{l}$ are the images of the points

$$
l \exp \left(\frac{2 \pi j(i-1)}{n}\right), \quad j=\sqrt{-1}, \quad(i=1,2, \ldots, n)
$$

being distributed on $\{w ;|w|=l\}$ under the mapping

$$
z=\frac{3 w}{2+w} \text {. }
$$

We consider the case when $l=1.9$ for (5.4). It is easily shown that the image of the charge points $\left\{z_{n, i}\right\}_{i=1}^{n}$ mapped by $\zeta=1 / z$ are uniformly distributed on

$$
\left\{\zeta ;\left|\frac{2}{1-3 \zeta}\right|=1.9\right\}
$$


(Fejér points) and so satisfy condition (A). It depends on the fact that

$$
H\left(\frac{1}{\zeta}\right)+\log |\zeta|=\log \left|\frac{1-3 \zeta}{2}\right|=\text { constant }
$$

implies

$$
\log \left|\frac{z-3}{2 z}\right|=\text { constant }
$$

The collocation points $\left\{\zeta_{n, i}\right\}_{i=1}^{n}$ on $\gamma$ are chosen for $l=1.0$ in (5.4).

For $n=10,11$ and the charge points with $l=1.9$, we solve a system of simultaneous linear equations (4.11) and (4.12) and obtain the following results (to know the distribution precisely, all the charges are denoted)

TABLE 1 . Charges for $n=10$ and $l=1.9$ by Inoue-Scheme.

\begin{tabular}{|l|l|l|l|l|l|}
\hline$\alpha_{0}$ & $0.40547084 E+00$ & $\alpha_{1}$ & $0.99539405 E-01$ & $\alpha_{2}$ & $0.10009742 E+00$ \\
\hline$\alpha_{3}$ & $0.10045396 E+00$ & $\alpha_{4}$ & $0.99316775 E-01$ & $\alpha_{5}$ & $0.10073638 E+00$ \\
\hline$\alpha_{6}$ & $0.99251514 E-01$ & $\alpha_{7}$ & $0.10073638 E+00$ & $\alpha_{8}$ & $0.99316775 E-01$ \\
\hline$\alpha_{9}$ & $0.10045396 E+00$ & $\alpha_{10}$ & $0.10009742 E+00$ & & \\
\hline
\end{tabular}

TABLE 2. Charges for $n=11$ and $l=1.9$ by Inoue-Scheme.

\begin{tabular}{|l|l|l|l|l|l|}
\hline$\alpha_{0}$ & $0.40546044 E+00$ & $\alpha_{1}$ & $0.99539405 E-01$ & $\alpha_{2}$ & $0.92778033 E-01$ \\
\hline$\alpha_{3}$ & $0.89046800 E-01$ & $\alpha_{4}$ & $0.92555946 E-01$ & $\alpha_{5}$ & $0.89742958 E-01$ \\
\hline$\alpha_{6}$ & $0.91332757 E-01$ & $\alpha_{7}$ & $0.91332757 E-01$ & $\alpha_{8}$ & $0.89742958 E-01$ \\
\hline$\alpha_{9}$ & $0.92555946 E-01$ & $\alpha_{10}$ & $0.89046800 E-01$ & $\alpha_{11}$ & $0.92778033 E-01$ \\
\hline
\end{tabular}

We note that $\log (3 / 2)=0.4054651 \ldots$ holds. We have the approximations $(4.14)$ as

$$
\alpha_{0} \simeq H(0)=\log \frac{3}{2}, \quad \alpha_{i} \simeq \frac{1}{n}, \quad(i=1,2, \ldots, n)
$$

with high accuracy for $n=10$ but for $n=11$.

This fact and Sugihara's paper [24] suggest to adopt the collocation points that satisfy also condition (A). Hence, we assume that the collocation points $\left\{\zeta_{n, i}\right\}_{i=1}^{n}$ are the images of the points (5.4) being distributed on $\{w ;|w|=1.0\}$ under the mapping

$$
z=\frac{3 w}{2+w} .
$$


TABLE 3. Charges for $n=10$ by Inoue-Scheme.

\begin{tabular}{|l|l|l|l|l|l|}
\hline$\alpha_{0}$ & $0.40562781 E+00$ & $\alpha_{1}$ & $0.99992591 E-01$ & $\alpha_{2}$ & $0.10001225 E+00$ \\
\hline$\alpha_{3}$ & $0.99983012 E-01$ & $\alpha_{4}$ & $0.10001133 E+00$ & $\alpha_{5}$ & $0.99995881 E-01$ \\
\hline$\alpha_{6}$ & $0.10000248 E+00$ & $\alpha_{7}$ & $0.99995881 E-01$ & $\alpha_{8}$ & $0.10001133 E+00$ \\
\hline$\alpha_{9}$ & $0.99983012 E-01$ & $\alpha_{10}$ & $0.10001225 E+00$ & & \\
\hline
\end{tabular}

TABLE 4 . Charges for $n=11$ by Inoue-Scheme.

\begin{tabular}{|l|c|c|c|c|c|}
\hline$\alpha_{0}$ & $0.40554302 E+00$ & $\alpha_{1}$ & $0.90884679 E-01$ & $\alpha_{2}$ & $0.90937077 E-01$ \\
\hline$\alpha_{3}$ & $0.90880650 E-01$ & $\alpha_{4}$ & $0.90935176 E-01$ & $\alpha_{5}$ & $0.90882271 E-01$ \\
\hline$\alpha_{6}$ & $0.90932148 E-01$ & $\alpha_{7}$ & $0.90882140 E-01$ & $\alpha_{8}$ & $0.90938645 E-01$ \\
\hline$\alpha_{9}$ & $0.90903198 E-01$ & $\alpha_{10}$ & $0.90885490 E-01$ & $\alpha_{11}$ & $0.90938532 E-01$ \\
\hline
\end{tabular}

It is known that these points are uniformly distributed on $\gamma_{1}$ [3]. By the same method as above, we obtain the results presented in Tables 3 and 4 .

Thus, we have the approximations (4.14) as

$$
\alpha_{0} \simeq H(0)=\log \frac{3}{2}, \quad \alpha_{i} \simeq \frac{1}{n}, \quad(i=1,2, \ldots, n)
$$

with high accuracy for $n=10$, and also for $n=11$.

The accuracy of the errors are estimated by

$$
\left|H_{n}\left(\zeta_{n, i+1 / 2}\right)-H\left(\zeta_{n, i+1 / 2}\right)\right|,
$$

where $\zeta_{n, i+1 / 2}$ is the middle point between $\zeta_{n, i}$ and $\zeta_{n, i+1}$.

By the Maximun Principle for the harmonic functions, it is sufficient that the errors are estimated only on the boundary.

For $n=10,11$ and the charge points with $l=1.9$ determined above (Tables 3 and 4 ), the errors are as follows (to know the distribution precisely, all the errors are denoted in Tables 5 and 6)

(5b) Using Inoue-Scheme and the charges $\left\{\alpha_{i}\right\}_{i=0}^{n}$ obtained by the same method as (5a) (Table 1 and 2), the approximations (4.13) are estimated to know the accuracy by increasing $l$ near the boundary and for a large $l$. The cases for $n=10,11$ and $l=1.1,1.3,1.5,1.7,1.9,10$ are studied. 
TABLE 5 . Errors for $n=10$ by Inoue-Scheme.

\begin{tabular}{|l|l|l|l|}
\hline $9.0822577 E-06$ & $7.1078538 E-06$ & $1.1324883 E-06$ & $4.7683715 E-07$ \\
\hline $3.5762786 E-07$ & $4.1723251 E-07$ & $5.3644180 E-07$ & $1.1622906 E-06$ \\
\hline $7.1078538 E-06$ & $9.0450048 E-06$ & & \\
\hline
\end{tabular}

TABLE 6 . Errors for $n=11$ by Inoue-Scheme.

\begin{tabular}{|l|l|l|l|}
\hline $1.9557774 E-06$ & $3.7103891 E-06$ & $6.2584877 E-07$ & $1.0728836 E-06$ \\
\hline $8.3446502 E-07$ & $1.1920929 E-07$ & $5.9604644 E-07$ & $1.3113021 E-06$ \\
\hline $8.6426734 E-07$ & $2.9057264 E-06$ & $1.9893050 E-06$ & \\
\hline
\end{tabular}

The accuracy of the errors are estimated by the same method as (5a) at the middle points $\zeta_{n, i+1 / 2}$ between $\zeta_{n, i}$ and $\zeta_{n, i+1}$. The maximums of $n$ errors for $n=10$ and 11 are represented in Table 7 .

TABLE 7. Maximum of $n$ errors for $n=10$ and 11 by Inoue-Scheme.

\begin{tabular}{|c|c|c|c|c|c|}
\hline 1.1 & 1.3 & 1.5 & 1.7 & 1.9 & 10 \\
\hline $4.9 E-03$ & $1.1 E-03$ & $2.8 E-04$ & $1.1 E-04$ & $5.7 E-05$ & $2.1 E-06$ \\
\hline $8.8 E-04$ & $6.7 E-04$ & $3.8 E-04$ & $3.3 E-04$ & $5.6 E-05$ & $2.7 E-05$ \\
\hline
\end{tabular}

(5c) Using Conv-Scheme, the accuracy of approximations will next be estimated to compare to Inoue-Scheme.

The charge points $\left\{z_{n, i}\right\}_{i=1}^{n}$ and the collocation points $\left\{\zeta_{n, i}\right\}_{i=1}^{n}$ are (5.4) for $l=$ $1.1,1.3,1.5,1.7,1.9,10$ and for $l=1.0$, respectively.

For $n=10,11$ and the charge points with each $l$, we solve a system of simultaneous linear equations by (4.15) and obtain the charges. Using the charges, we estimate the errors $H_{n}(z)-H(z)$ by the same method as (5b).

(5d) Using Murota-Scheme, the accuracy of approximations will next be estimated to compare to Inoue-Scheme and Conv-Scheme.

The charge points $\left\{z_{n, i}\right\}_{i=1}^{n}$ and the collocation points $\left\{\zeta_{n, i}\right\}_{i=1}^{n}$ are also (5.4) for $l=1.1,1.3,1.5,1.7,1.9,10$ and for $l=1.0$, respectively. 
TABLE 8. Maximum of $n$ errors for $n=10$ and 11 by Conv-Scheme.

\begin{tabular}{|c|c|c|c|c|c|}
\hline 1.1 & 1.3 & 1.5 & 1.7 & 1.9 & 10 \\
\hline $6.7 E-01$ & $3.5 E-01$ & $8.6 E-03$ & $3.1 E-03$ & $1.4 E-03$ & $3.8 E-04$ \\
\hline $4.1 E-01$ & $2.6 E-02$ & $5.6 E-03$ & $1.9 E-03$ & $7.8 E-04$ & $2.7 E-04$ \\
\hline
\end{tabular}

TABLE 9. Maximum of $n$ errors for $n=10$ and 11 by Murota-Scheme.

\begin{tabular}{|c|c|c|c|c|c|}
\hline 1.1 & 1.3 & 1.5 & 1.7 & 1.9 & 10 \\
\hline $1.3 E-01$ & $2.8 E-02$ & $3.2 E-03$ & $3.3 E-03$ & $1.5 E-03$ & $3.7 E-04$ \\
\hline $1.1 E-01$ & $2.1 E-02$ & $5.6 E-03$ & $2.0 E-03$ & $8.3 E-04$ & $2.5 E-04$ \\
\hline
\end{tabular}

For $n=10,11$ and the charge points with each $l$, we solve a system of simultaneous linear equations by (4.16) and (4.17) and obtain the charges. Using the charges, we estimate the errors $H_{n}(z)-H(z)$ by the same method as (5b).

(5e) Using Fundamental Lemma with mathematical scheme (4.6), the accuracy of approximations will last be estimated to compare to the above errors in (5a)-(5d).

The charge points $\left\{z_{n, i}\right\}_{i=1}^{n}$ are same as (5a). The collocation points $\left\{\zeta_{n, i}\right\}_{i=1}^{n}$ are not used.

For $n=10,11$, we apply Algorithm 4.1 with the charge at every charge point being $1 / n$. We need not solve a system of simultaneous linear equations.

We estimate the errors $H_{n}(z)-H(z)$ by the same method as (5b).

TABLE 10. Errors for $n=11$ and $l=10$, by Math-Scheme at middle points.

\begin{tabular}{|l|l|l|l|}
\hline $1.8626451 E-08$ & $2.9802322 E-08$ & $2.9802322 E-08$ & $0.0000000 E+00$ \\
\hline $5.9604644 E-08$ & $5.9604644 E-08$ & $1.1920929 E-07$ & $0.0000000 E+00$ \\
\hline $0.0000000 E+00$ & $1.4901161 E-08$ & $8.9406967 E-08$ & \\
\hline
\end{tabular}

It is necessary to estimate the errors at the collocation points in (5a) for this case.

6. Concluding remark. Some remarks on the above numerical experiments will be given in this section

(6a) In all the cases, the accuracy is not improved by increasing the distance of the charge points from the boundary when they are not too near to the boundary.

(6b) The accuracy of (5e) is superior to (5b), (5c) and (5d). By the new scheme introduced in this paper, numerical results of higher accuracy than the conventional one are obtained. 
TABLE 11 . Errors for $n=11$ and $l=10$ by Math-Scheme at collocation points.

\begin{tabular}{|l|l|l|l|}
\hline $1.4901161 E-08$ & $2.2351741 E-08$ & $5.9604644 E-08$ & $1.1920929 E-07$ \\
\hline $1.7881393 E-07$ & $0.0000000 E+00$ & $0.0000000 E+00$ & $0.0000000 E+00$ \\
\hline $1.7881393 E-07$ & $2.9802322 E-08$ & $2.9802322 E-08$ & \\
\hline
\end{tabular}

(6c) The difference of the accuracy cannot be found between even $n$ and odd $n$ in the above cases.

(6d) Numerical experiments show that the approximations are impossible to get for a small $l-1$ (for example $l=1.01$ ) in (5c) and (5d). However, the errors for $n=10$ and $l=1.01$ in $(5 \mathrm{e})$ are as follows

TABLE 12. Errors for $n=10$ and $l=1.01$ by Math-Scheme at middle points.

\begin{tabular}{|l|l|l|l|}
\hline $9.4208717 E-03$ & $4.7457519 E-03$ & $5.8910250 E-04$ & $7.2479248 E-05$ \\
\hline $4.8339366 E-05$ & $4.8279762 E-05$ & $7.2658061 E-05$ & $5.8916211 E-04$ \\
\hline $4.7457665 E-03$ & $9.4208717 E-03$ & & \\
\hline
\end{tabular}

TABLE 13 . Errors for $n=10$ and $l=1.01$ by Math-Scheme at collocation points.

\begin{tabular}{|l|l|l|l|}
\hline $1.6323477 E-04$ & $4.0158629 E-05$ & $4.5597553 E-06$ & $5.9604644 E-07$ \\
\hline $0.0000000 E+00$ & $1.7881393 E-07$ & $1.1920929 E-07$ & $5.9604644 E-07$ \\
\hline $4.5001506 E-06$ & $4.0069222 E-05$ & & \\
\hline
\end{tabular}

(6e) The distribution of the charge points for (5e) is quite different from the conventional one. More precisely, the circle $\{z ;|z-25 / 8|=5 / 8\}(l=10)$ containing all the charge points does not bound the domain $D$.

(6f) The main object of this paper is to consider the fundamental solutions method mathematically. Therefore, the accuracy has been estimated for $n=10,11$ and by simple precision (Runfor $f-77, P C-486 A V$ ). The numerical experiments for $n=20,30, \ldots$ and by bouble precision will appear in a future paper.

(6g) The new method, especially (5e), introduced in this paper gives higher accuracy than the conventional one.

(6h) The conventional method is superior in the sense that it can be applied to any harmonic function with a same distribution of the charge points and the regularity of the coefficient matrix is verified for the domain with an analytic boundary data. 
(6i) Though the numerical examlpes have been shown only for bounded Jordan domains, the analogous results can be obtained also for unbounded Jordan domains.

We wish that the method mathematically introduced in this paper would contribute to the developement of conventional fundamental solutions method.

\section{REFERENCES}

[1] L. V. Ahlfors, Complex analysis: An introduction of the theory of analytic functions of one complex variable, 2nd ed., vol. 48, McGraw-Hill Book Co., New York, Toronto, London, 1966. MR 32\#5844. Zbl 154.31904.

[2] K. Amano, A charge simulation method for the numerical conformal mapping of interior, exterior and doubly-connected domains, J. Comput. Appl. Math. 53 (1994), no. 3, 353-370. MR 95i:30007. Zbl 818.30004.

[3] D. Gaier, Lectures on complex approximation, Birkhäuser Boston, Inc., Boston, 1987, (Translated from the German by Renate McLaughlin). MR 88i:30059b. Zbl 612.30003 .

[4] T. Inoue, A characterization of weighted Chebyshev constant, Appl. Math. Lett. 5 (1992), no. 2, 71-74. MR 92k:41015. Zbl 766.41008.

[5] _ Asymptotic behavior of extremal weighted polynomials, Mathematica (Cluj) 35 (1993), no. 1, 29-34. MR 95k:30010. Zbl 803.30005.

[6] _ Applications of asymptotic theorem on weighted extremal polynomials, Trans. Japan Soc. Ind. App. Math. 4 (1994), 151-155 (Japanese).

[7] _ Equilibrium measure and limit zeros distribution of extremal weighted polynomials, Appl. Math. Lett. 7 (1994), no. 4, 75-78. CMP 96 01. Zbl 814.30004.

[8]__ Fundamental propositions on weighted polynomials, Mathematica (Cluj) 36 (1994), no. 1, 33-35. MR 96f:31002. Zbl 862.30025.

[9]___ Asymptotic theorem on weighted extremal polynomials and its application for theoretical approach to charge simulation method, Conference Japan Ind. Appl. Math. Soc. (1995), 26 (Japanese).

[10] _ Some properties of weighted hyperbolic polynomials, Internat. J. Math. Math. Sci. 19 (1996), no. 1, 9-14. MR 96k:30037. Zbl 844.30027.

[11]__ Zeros distribution of normalized counting measures, Mathematica (Cluj) 38 (1996), 67-73. Zbl 873.31003.

[12] M. Katsurada, A mathematical study of the charge simulation method. II, J. Fac. Sci. Univ. Tokyo Sect. IA Math. 36 (1989), no. 1, 135-162. MR 90d:35061. Zbl 681.65081.

[13] _ Asymptotic error analysis of the charge simulation method in a Jordan region with an analytic boundary, J. Fac. Sci. Univ. Tokyo Sect. IA Math. 37 (1990), no. 3, 635-657. MR 91m:65263. Zbl 723.65093.

[14] M. Katsurada and H. Okamoto, The collocation points of the fundamental solution method for the potential problem, Comput. Math. Appl. 31 (1996), no. 1, 123-137. CMP 96 04. Zbl 852.65101.

[15] N. S. Landkof, Foundations of modern potential theory, Springer-Verlag, New York, Heidelberg, 1972, Translated from the Russian by A. P. Doohovskoy. MR 502520. Zbl 253.31001.

[16] H. N. Mhaskar, Finite-infinite range inequalities in the complex plane, Internat. J. Math. Math. Sci. 14 (1991), no. 4, 625-638. MR 93b:26023. Zbl 774.30004.

[17] H. N. Mhaskar and E. B. Saff, Where does the sup norm of a weighted polynomial live?, Constr. Approx. 1 (1985), no. 1, 71-91, (A generalization of incomplete polynomials). MR 86a:41004. Zbl 582.41009.

[18] _ The distribution of zeros of asymptotically extremal polynomials, J. Approx. Theory 65 (1991), no. 3, 279-300. MR 92d:30005. Zbl 744.41007.

[19] _ Weighted analogues of capacity, transfinite diameter, and Chebyshev constant, Constr. Approx. 8 (1992), no. 1, 105-124. MR 93a:31004. Zbl 747.31001. 
[20] S. Murasima, Charge Simulation Method and its Application, Morikita Syuppan, Tokyo (1983) (Japanese).

[21] K. Murota, On "invariance" of schemes in the fundamental solution method, Trans. Inform. Process. Soc., Japan 3 (1993), 533-535 (Japanese).

[22] _ Comparison of conventional and "invariant" schemes of fundamental solutions method for annular domains, Japan J. Indust. Appl. Math. 12 (1995), no. 1, 61-85. MR 95k:65098. Zbl 831.65118.

[23] K. Nishida, Mathematical and numerical analysis of charge simulation method in 2dimensional elliptic domains, Trans. Japan Soc. Ind. App. Math. 5 (1995), 185-198 (Japanese), Dissertation, Tokyo Univ. (1995).

[24] M. Sugihara, Approximations of harmonic functions, Koukyuroku 676, Research Inst. Math. Sci. Kyoto Univ. (1988), 251-261 (Japanese).

[25] M. Tsuji, Potential theory in modern function theory, Chelsea Publishing Co., New York, 1975, Reprinting of the 1959 original. MR 54 2990. Zbl 322.30001.

[26] S. Yotsutani, The distribution of charge points in charge simulation method, Koukyuroku 703, Research Inst. Math. Sci. Kyoto Univ. (1989), 172-186 (Japanese).

INOUE: DEPARTMENT OF INFORMATION SYSTEMS ENGINEERING, KOBE UNIVERSITY OF MERCANTILE MARINE, KOBE, JAPAN

E-mail address: inoue-te@ti edu.kshosen.ac.jp 


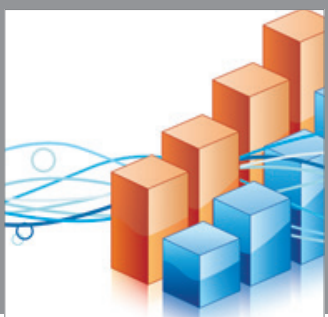

Advances in

Operations Research

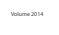

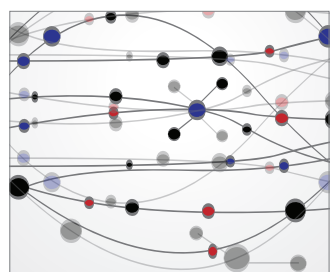

\section{The Scientific} World Journal
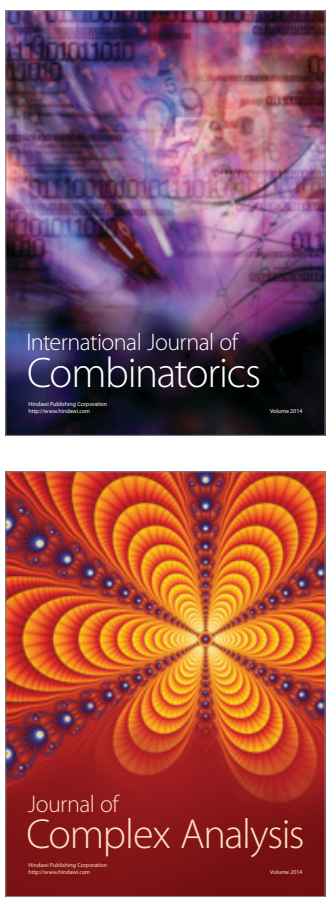

International Journal of

Mathematics and

Mathematical

Sciences
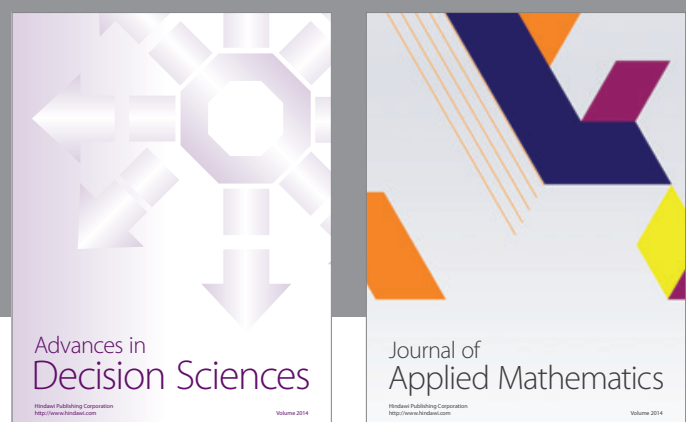

Journal of

Applied Mathematics
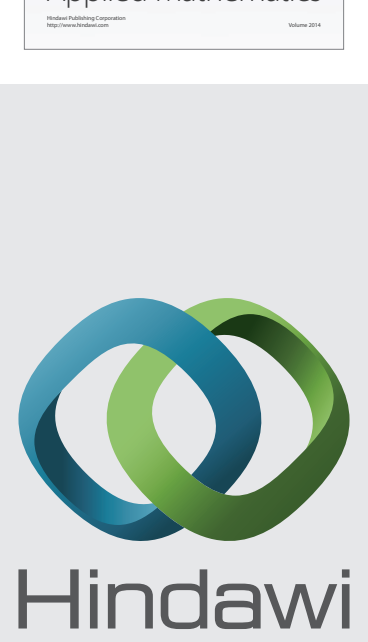

Submit your manuscripts at http://www.hindawi.com
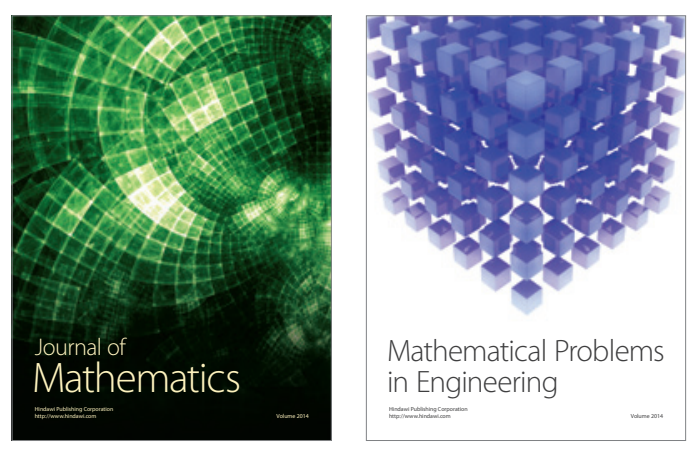

Mathematical Problems in Engineering
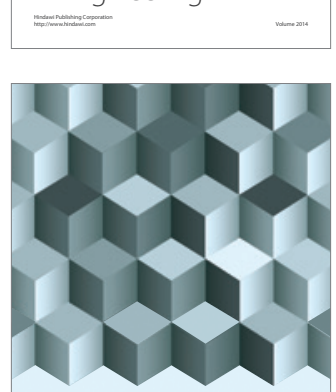

Journal of

Function Spaces
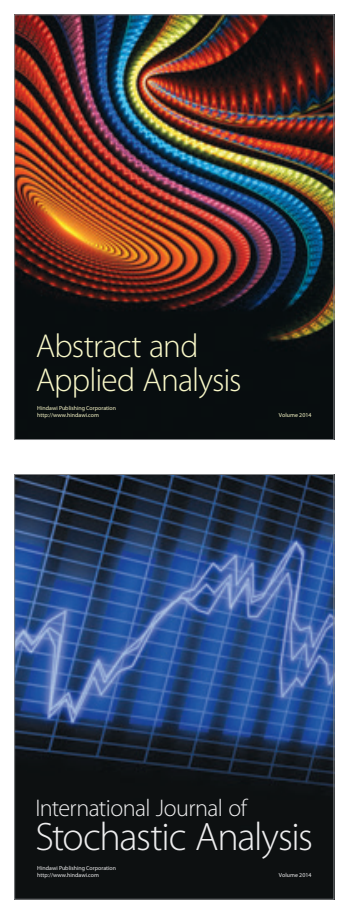

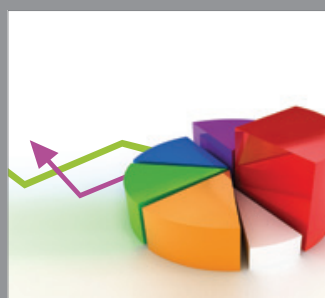

ournal of

Probability and Statistics

Promensencen
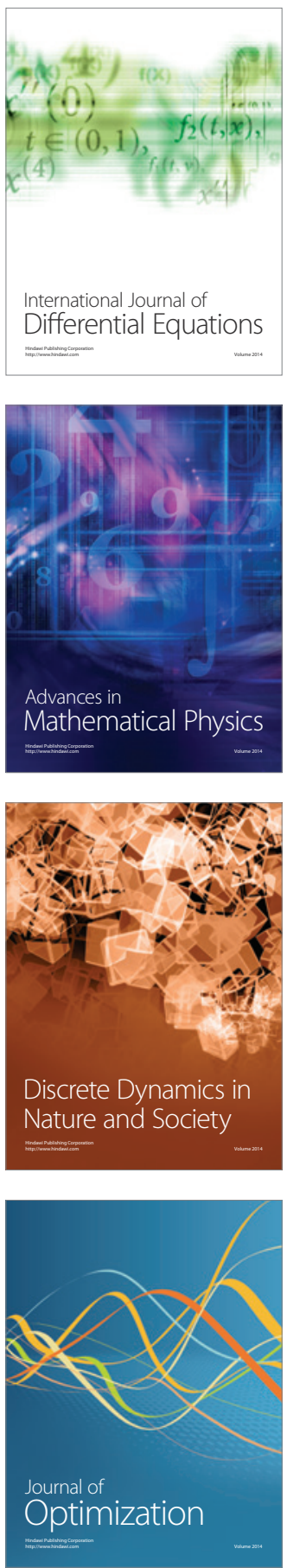